\title{
Everything is possible if we work together
}

Three transcription factors - OCT4, SOX2 and NANOG - are essential for maintaining the pluripotency of human embryonic stem (ES) cells. Young and colleagues have now identified the target genes of these three proteins, and have uncovered several regulatory circuits through which they fulfil their function.

Using a combination of chromatin immunoprecipitation and DNA microarrays, the authors assayed the regions near the promoters of 17,917 annotated human genes. They found that OCT4, SOX2 and NANOG bound near the promoters of 623 , 1,271 and 1,687 genes, in that order. What was particularly surprising was that at least 353 of those genes were bound by all three transcription factors, which implies a significant coordination of function.

About half these coordinately regulated genes are activated by OCT4, SOX2 and NANOG, and half are repressed. The activated genes include components of the TGFB and WNT signalling pathways, both of which have a role in maintaining pluripotency; the repressed genes include many transcription factors that are important for differentiation.

The authors confirmed the presence of two types of regulatory circuit in human ES cells, using algorithms that had previously been devised in yeast. First, they found feed-forward loops, in which a first regulator regulates a second regulator and both then regulate the target genes.
If both regulatory steps are positive this gives stability against transient changes in input; if one step is negative a rapid switch in response to changed conditions is enabled.

The second type of circuitry that they describe is the autoregulatory loop, in which OCT4, SOX2 and NANOG regulate their own expression. This also offers stability of gene expression and rapid responses to environmental stimuli.

These approaches will allow researchers to elucidate the circuits that are controlled by other transcription factors, and also by chromatin regulators. Testing these circuits will be aided by advances in the culture and genetic manipulation of ES cells. A full understanding of the regula-

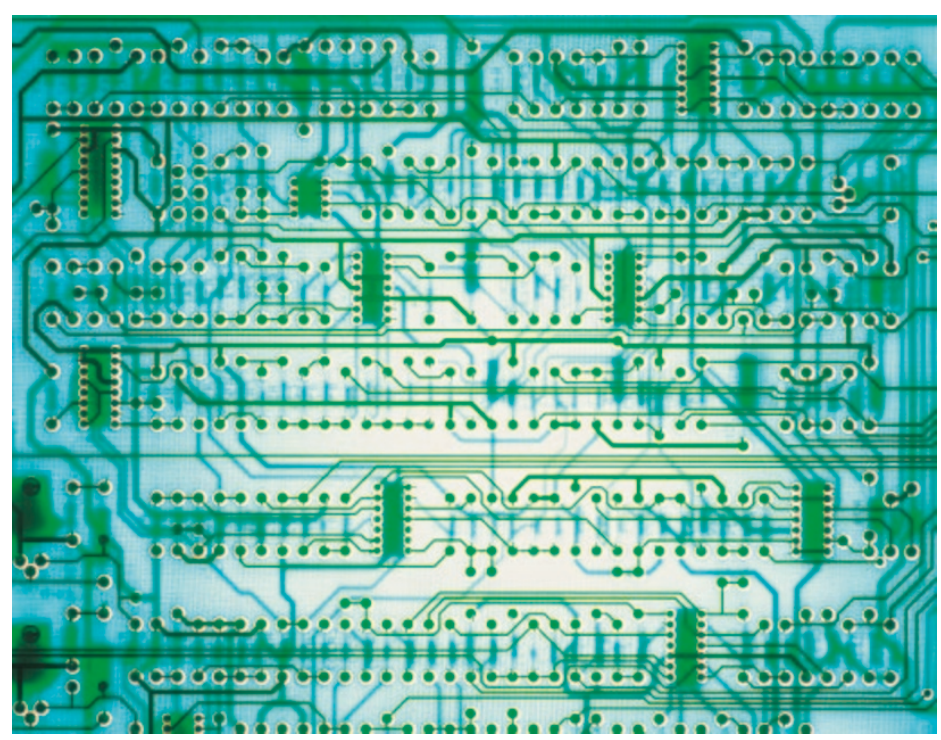

tory circuitry of ES cells will help researchers to promote stem cells to differentiate into a range of cell types, and possibly to reprogramme differentiated cells back into pluripotent ones.

Patrick Goymer

\section{(1) References and links}

ORIGinal ReSEARCh PAPER Boyer, L. A. et al. Core transcriptional regulatory circuitry in human embryonic stem cells. Cell 122, 1-10 (2005) WEB SITE

Richard A. Young's laboratory: http://web. wi.mit.edu/young 